\title{
Model Reduction of a Load-Sensing Hydraulic System via Activity Index Analysis
}

\author{
Quanyi Hu1,* - Hong Zhang1 - Shujun Tian ${ }^{1}$ - Xuxin Qin² \\ 1 Dalian University of Technology, School of Mechanical Engineering, China \\ 2 Linde Hydraulics GmbH \& Co. KG, Germany
}

\begin{abstract}
A load-sensing hydraulic system is an energy-saving fluid power transmission that supplies "on-demand" flow at a prescribed pressure margin greater than load pressure. In the paper, based on bond graph methodology, a complex and non-linear load-lensing (LS) hydraulic system model is constructed, in which the dynamic behaviours of an LS valve are taken into account. Moreover, a series of experiments are performed to validate the model. Two reduced models based on activity index analysis are obtained to achieve a balance between accuracy and complexity. The results indicate that the system order and computation time can be reduced. By quantifying the relative importance of the elements based on activity index analysis, it shows that the inner leakage of the pump plays a major role in system dynamics due to its correlation to the damping coefficient. Furthermore, the results prove the rationality of representing LS pump dynamics with a fourth-order differential equation and the fact that the dynamics of $L S$ valves have not been well-covered in the literature.
\end{abstract}

Keywords: load-sensing, bond graph, modelling, model reduction, activity index, MORA

Highlights

- $\quad$ Bond graph modelling of the load-sensing hydraulic system.

- $\quad$ Valid bond graph model verified by a series of experiments.

- $\quad$ Two reduced models achieving a balance between accuracy and complexity.

- $\quad$ The rationality representing the LS pump dynamic with a fourth-order differential equation.

\section{O INTRODUCTION}

A Load-Sensing hydraulic system (LS) is an energysaving fluid power transmission that has been widely used in mobile hydraulic applications [1] and [2], such as excavator, material handling, and agricultural machines, etc. Furthermore, the combination of electronics and LS system can offer new solutions for accurate motion control.

In principle, the LS system supplies "on-demand" flow at a prescribed pressure margin greater than the highest load pressure and eliminates the bypass losses due to the closed centre. However, the LS system is prone to oscillation, and sometimes becomes unstable due to the feedback of the load-sensing mechanism. Furthermore, as presented by Backe [3] and Kim [4], it is also difficult to obtain a satisfactory overall performance because a contradiction exists between energy efficiency and rapid dynamic response, especially over a wide range of operating conditions. Thus, the efforts for the design of the LS system are in general significantly more than that for the conventional hydraulic system with constant delivery pressure or constant delivery flow rate.

To obtain better insight into the performance characteristics of the LS system, a mathematical model is needed. Up to now, considerable research has been conducted into the modelling of the LS system. Most of them used simplified mathematical models with a fourth-order differential equation for the LS pump and ignored the dynamic effects of the LS valve and the cut-off valve. In the literature, Krus [5], Jayaraman [6], Axin [7] and Xu [8] provided a detailed model of an LS system based on transfer function and a block diagram. Koivumaki and Mattila [9] presented a nonlinear model based on a motion control scheme. Wagner [10] and $\mathrm{Wu}$ [11] presented a stability analysis in the frequency domain, but the model did not consider the dynamic of the LS valve and the LS pump was simplified with a second-order system. Sakurai [12] and Sakurai et al. [13] proposed a design method for the intelligent LS system and studied the computation of the overall efficiency by a simple bond graph model, which neglected the dynamic of the LS pump.

From the viewpoint of modelling, the LS system is essentially a complex, highly nonlinear system which comprises interacting components from mechanical, electrical, hydraulic and control subsystems. Compared to other modelling methods such as block diagram, signal flow graph, etc., bond graph methodology [14] to [16] is a better graphical representation suitable for modelling the multidisciplinary dynamic engineering system. As a core representation, the bond graph model can preserve the computational and topological structure. Moreover, it 
can be systematically converted into state-equations, transfer functions, block diagrams and signal flow graphs, etc.

In general, the proper model should be as simple as possible for a particular application. However, the modeling procedure often ends with an excessively complex model. Compared to the complex model, a reduced model has several advantages. Firstly, the reduced model is generally a lower-order system, which is conducive to controller design, because the lower-order controller is easier to implement. Secondly, the reduced model can achieve a balance between model accuracy and computation speed, which is crucial for applications requiring both accuracy and efficient computation, such as system optimization and hardware-in-the-loop (HIL) simulation which needs real-time computation. Furthermore, the reduced model is easier to inspect and interpret from physical insights. Finally, reduced model is tractable for the lower number of parameters needed to be identified in system identification and optimization.

The literature for model reduction can be classified into frequency, projection, optimization, and energy-based [17]. Among them, the energybased model reduction technology is particularly suitable for bond graph models due to the common energy-based nature. The Rayleigh-Ritz methodology [17] exemplifies the core metric for quantifying the relative importance of the elements for a given system. Rosenberg and Zhou [18] proposed a powerbased model reduction algorithm. Louca et al. [19]
[20] extended Rosenberg and Zhou's algorithm by proposing a new energy-based model reduction metric called activity which avoids the immediate and timevarying decisions about the importance of elements, subsequently, an activity-based model order reduction algorithm (MORA) is proposed.

In this paper, based on the bond graph methodology, an accurate but overly complex LS system model taking into account the dynamic characteristics of the LS valve and the cut-off valve is constructed. Moreover, a series of experiments are performed to validate the bond graph model. Then, based on the activity index analysis, two reduced models with different thresholds are obtained directly from the bond graph level. Thus, a balance between accuracy and complexity is achieved. Furthermore, by quantifying the relative importance of the elements based on the activity index analysis, it proves the rationality representing LS pump dynamics with the fourth-order differential equation in the literature. Finally, the effects of the user-defined threshold on the model accuracy are explored.

\section{PRINCIPLE OF THE LS SYSTEM}

As shown in Fig. 1, the LS system driving a simple inertial load with damping resistance is illustrated.

In Fig. 1, the heart of the LS system is the LS pump, which is often a swash-plate-type variable displacement piston pump integrating an LS valve and a cut-off valve. The LS valve is essentially a pressureflow compensator, which compares pump delivery

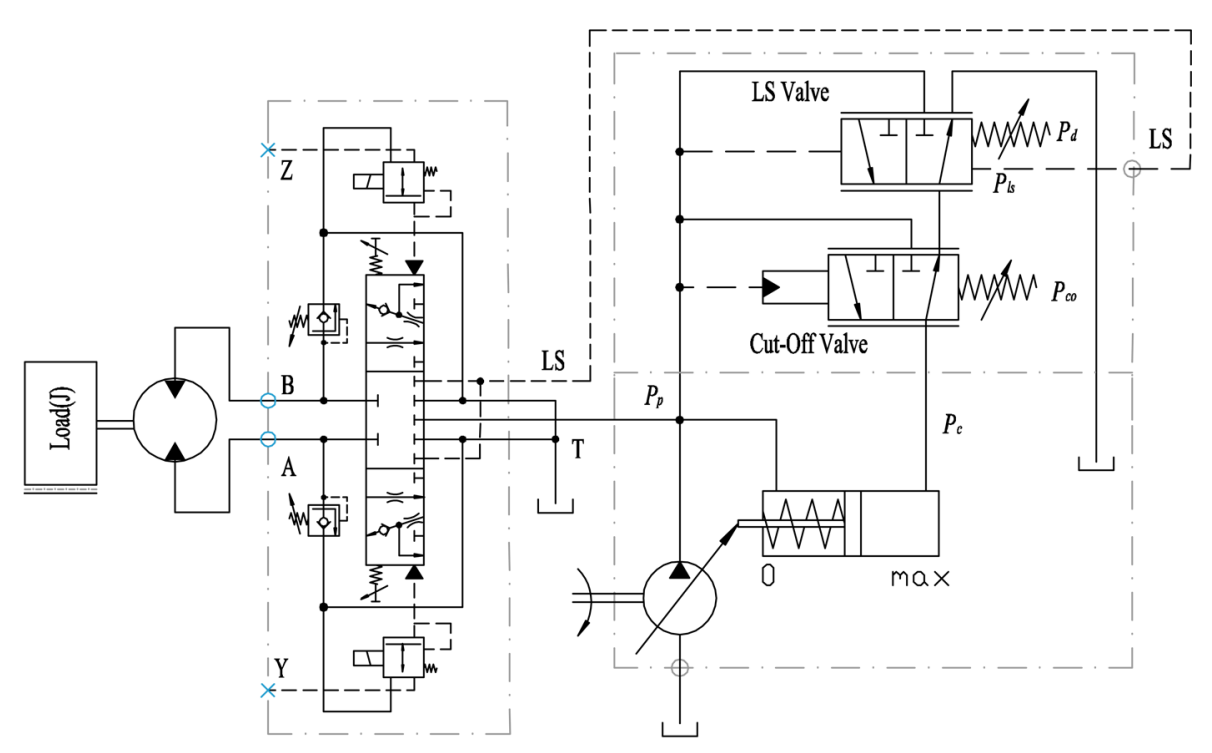

Fig. 1. Schematic of the LS system 
pressure $P_{p}$ with load pressure $P_{l s}$ transmitted through the LS port of the directional valve. The operation principles of the LS system are described in detail as follows:

When the directional valve is positioned at the certain opening, the LS valve will sense the pressure difference of pump delivery pressure $P_{p}$ and load pressure $P_{l s}\left(\Delta P=P_{p}-P_{l s}\right)$, and the position of the spool is determined by the acting force generated by the pressure difference $\Delta P$ and the initial precompression force of the LS spring. The setting value of the LS spring determines the prescribed pressure margin $P_{d}$. When the pressure difference $\Delta P$ is smaller than the prescribed pressure margin $P_{d}$, the spool of the LS valve will be positioned at the right side, as shown in Fig. 1; the pressure oil in the displacement control piston will be released to the reservoir through the cut-off valve and the LS valve; thus the pump displacement will increase. In contrast, when the pressure difference $\Delta P$ is larger than the prescribed pressure margin $P_{d}$, the pump delivery oil will flow into the displacement control piston; thus, the pump displacement will decrease. Consequently, the pressure difference $\Delta P$ is regulated to be retained constant, approximately equal to the prescribed pressure margin $P_{d}$.

In addition, the volumetric flow through the directional valve at a given opening can be assessed using Eq. (1):

$$
Q_{v}=C_{d} \times A \times \sqrt{\frac{2 \times \Delta P}{\rho}},
$$

where $Q_{v}$ is volumetric flow rate, $C_{d}$ is flow coefficient of directional valve, and $\rho$ is the density of the oil.

As indicated by Eq. (1), due to the approximately constant pressure difference $\Delta P$, the delivery volumetric flow from the pump is only related to the opening of the directional valve, irrespective of the load pressure, which leads to an "on demand" flow at a prescribed pressure margin higher than the load pressure.

When the LS system is running in an idle condition, the directional valve will be closed, and the LS valve will de-stroke the pump displacement until the output flow is nearly zero, which should be sufficient for covering system leakages and for heat removal. Moreover, the pump delivery pressure goes up to the standby pressure, which is decided by the prescribed pressure margin $P_{d}$. The standby pressure is approximately $0.3 \mathrm{MPa}$ higher than the prescribed pressure margin, which is caused by the friction and flow forces acting on the LS spool.

The maximum pump delivery pressure is set for safety by a cut-off valve which will de-stroke the pump displacement when the pump delivery pressure is over the setting value $P_{c o}$.

\section{SYSTEM MODELLING BASED ON BOND GRAPH}

The LS system is essentially a complex, highly nonlinear system. This section starts from bond graph principles, and then develop the LS model. Finally, a series of experiments are carried out to validate the model.

\subsection{Bond Graph Modelling Principles}

The bond graph was proposed by Paynter in 1959 [14]. It is a unified modelling method that is particularly suited for modelling multi-disciplinary engineering systems.

The bond graph methodology established a group of unified variables, $\{e(t), f(t), p(t), q(t)\}$, associated with power and energy to model the physical systems. Among them, the effort $e(t)$ and flow $f(t)$ are power variables; the momentum $p(t)$ and displacement $q(t)$ are energy variables.

The power $P(t)$ and energy $E(t)$ flowing into or out of a port can be expressed as Eq. (2) and Eq. (3), respectively.

$$
\begin{gathered}
P(t)=e(t) \cdot f(t) \\
E(t)=\int^{t} e(t) f(t) d t=\int^{q} e(q) d q=\int^{p} f(p) d p .
\end{gathered}
$$

The bond graph model represents the physical processes in which energy is distributed, transferred and transformed between interconnected ports from different domains with the basic elements $\{R, C, I$, $\left.S_{e}, S_{f}, T F, G Y, 0,1\right\}$. Inertance " $P$ ", capacitance " $C$ ", resistance $R$, flow source " $S_{f}^{\prime}$ ", and effort source " $S_{e}$ " represent the physical phenomena which have the definite constitutive relations. Junction structures are assemblages of " 0 " and " 1 " junctions, transformer " $T F$ ", and gyrator " $G Y$ ", which enforce the energy conservation constraints among parts of the system. Furthermore, it reflects the topological structure of the physical system.

Bonds denote point-to-point connections between power ports of the subsystems or elements where energy flows into or out of the ports, as shown in Fig. 2a, A and B represent the subsystem or element. The harpoon indicates the instantaneous power flow 
direction. Each bond simultaneously brings a pair of conjugated variables, effort $e(t)$ and flow $f(t)$, where the product gives the transmitted power.

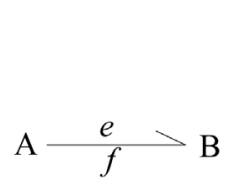

a)

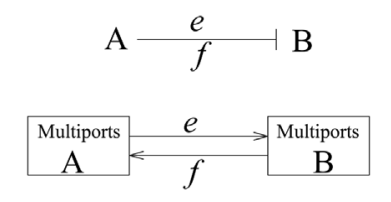

b)
Fig. 2. Power bond; a) power direction, b) causality assignment

When formulating the system equations for simulation, causality plays a key role which represents the computational structure of the model. The assigned causality dictates which port variable will be computed as a result, and consequently, the other port variable will be the cause. In the bond graph, the causal stroke in a bond indicates the direction in which the effort variable is directed, as shown in Fig. $2 b$.

After developing the casual bond graph model, it is straightforward to derive system equations in an orderly fashion, and then to obtain the numerical solutions of the conjugated variables, effort $e(t)$ and flow $f(t)$, on all bonds by many available numerical solvers.

\subsection{LS System Modelling}

As shown in Fig. 1, the LS system consists of the LS pump, directional valve, fixed displacement motor and inertial load with damping resistance.

\subsubsection{LS Pump Modelling}

As shown in Fig. 1, the LS pump is a pressure-flow compensated variable displacement pump which is often a swash-plate-type axial piston pump integrating the LS valve and cut-off valve.

Fig. 3a shows the physical structure of the swashplate-type piston pump that comprises the rotating components and the adjusting mechanism for control of pump displacement [21] and [22]. The pump displacement is a function of the inclination angle $\alpha$ of the swash-plate that is controlled by the control piston, the bias piston, and the return spring.

The governing equation describing the motion of the swash-plate can be written as Eq. (4).

$$
J_{p} \ddot{\alpha}+B_{p} \dot{\alpha}+\left(G_{p}-R\right) \alpha=D_{b} P_{p}-D_{c} P_{c}+G_{p} \alpha_{0},
$$

where $\alpha$ is the angle of the swash-plate, and $P_{p}, P_{c}$ is the pressure of pump output and LS valve outlet, respectively.

The rotating components can be represented by a controlled flow source $M S_{f}$, and the pump displacement is controlled by the inclination angle $\alpha$ of the swash-plate. The torque generation on the swash-plate is a function of the geometrical data of the pump and the operating conditions. The flow continuity into the pump discharge chamber and the displacement control piston are both represented by " 0 " junction. The bond graph model of the LS pump is shown in Fig. 3b.

In the model, the torque coefficient $(G-R)$ is represented by an equivalent capacitance element $K_{p}$.

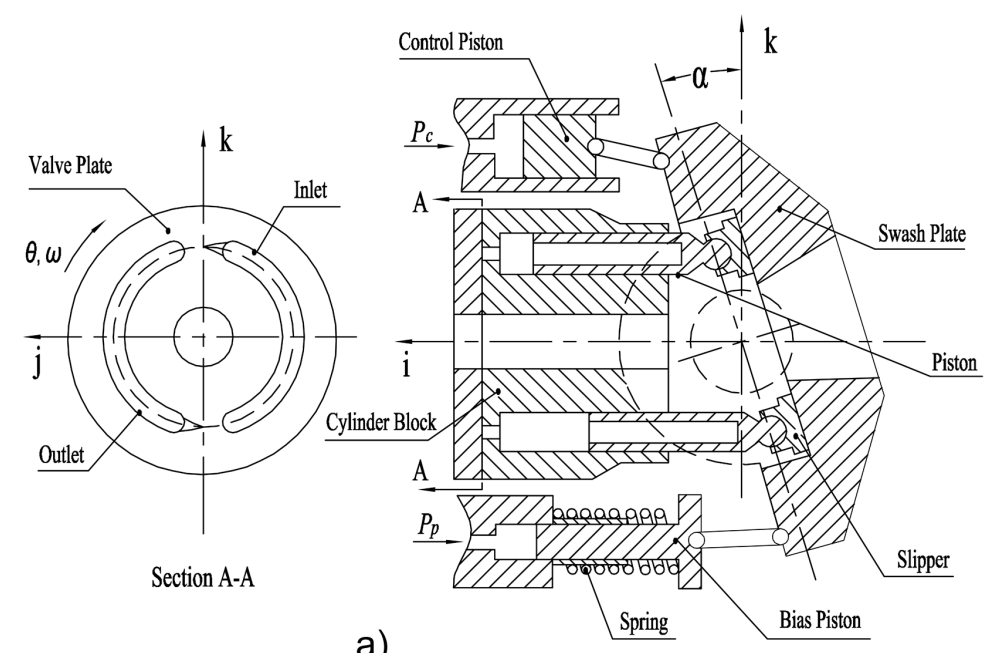

a)

Fig. 3. LS pump modelling; a) physical structure, b) bond graph model

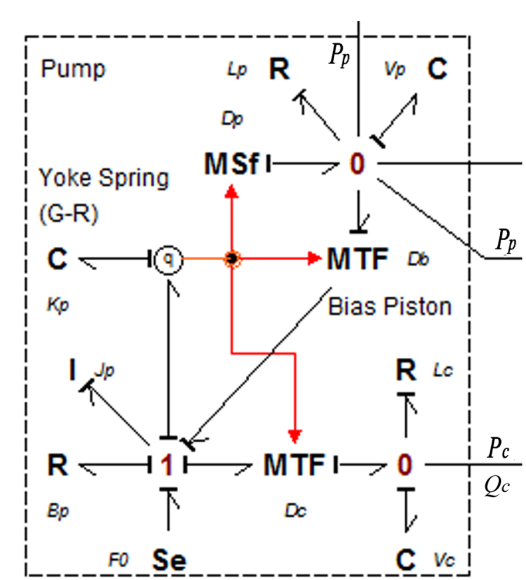

b) 
The initial setting of the return spring is described with an effort source $F_{0}$.

\subsubsection{LS valve and Cut-off Valve Modelling}

The LS valve is devoted to sensing the pressure difference $\Delta P$ between pump delivery pressure $P_{p}$ and load pressure $P_{l s}$, the position of the spool is regulated by the acting force generated by the pressure difference $\Delta P$ and the initial pre-compression force of the LS spring. The cut-off valve is set for safety by destroking the pump when the pump delivery pressure is over the setting value. The physical structure of the LS valve and the cut-off valve is shown in Fig. 4a.

The volumetric flow through the LS valve and the cut-off valve can be expressed in Eq. (5) and Eq. (6).

$$
\begin{gathered}
Q_{l s}=\left\{\begin{array}{cc}
K_{M R 3}\left(P_{l s}+P_{d}-P_{p}\right) \sqrt{P_{l s}} & P_{l s}+P_{d}>P_{p} \\
K_{M R 4}\left(P_{l s}+P_{d}-P_{p}\right) \sqrt{P_{p}-P_{l s}} & P_{l s}+P_{d} \leq P_{p}
\end{array},\right. \\
Q_{c}=\left\{\begin{array}{cc}
K_{M R 1}\left(P_{p}-P_{c o}\right) \sqrt{P_{p}-P_{c}} & P_{p}>P_{c o} \\
K_{M R 2}\left(P_{p}-P_{c o}\right) \sqrt{P_{c}} & P_{p} \leq P_{c o}
\end{array}\right.
\end{gathered}
$$

where $Q_{l s}, Q_{c}$ are flows to LS valve and cut-off valve, respectively, $P_{d}, P_{c o}$ are the setting pressure of LS valve and cut-off valve, respectively, and $P_{l s}$ is load pressure from the directional valve.
Fig. $4 \mathrm{~b}$ shows the bond graph model of the LS valve and the cut-off valve. In the model, the dynamic effects in the LS valve and the cut-off valve are considered, including inertia, resistance, and capacitance.

\subsubsection{Full LS System Modelling}

Based on the above component modelling, an accurate but overly complex bond graph model of the LS system is constructed, as shown in Fig. 5.

As shown in Fig. 5, an electro-proportional directional valve integrating an LS port to transmit the load pressure is used in the system. The dynamics of the valve can be considered as an equivalent firstorder model since its dynamics are much faster than that of the pump and load dynamics, the equation can be written as

$$
\tau \dot{x}_{v}+x_{v}=K i
$$

where $x_{v}$ is the opening of the directional valve, and $i$ is an input signal.

As for the fixed displacement piston motor, the inner leakage is considered by coefficient $L_{m}$, the capacitance effect between the directional valve and motor are considered by $V_{m 1}$ and $V_{m 2}$, respectively.

Generally, the built model is generic, and it can be used for the simulation of various kinds of LS system so long as the schematic is similar to this.

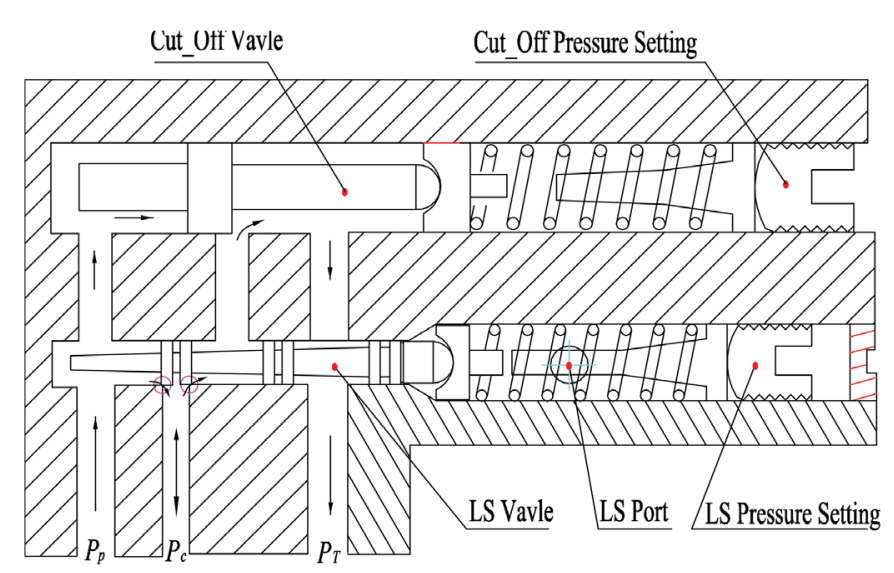

a)

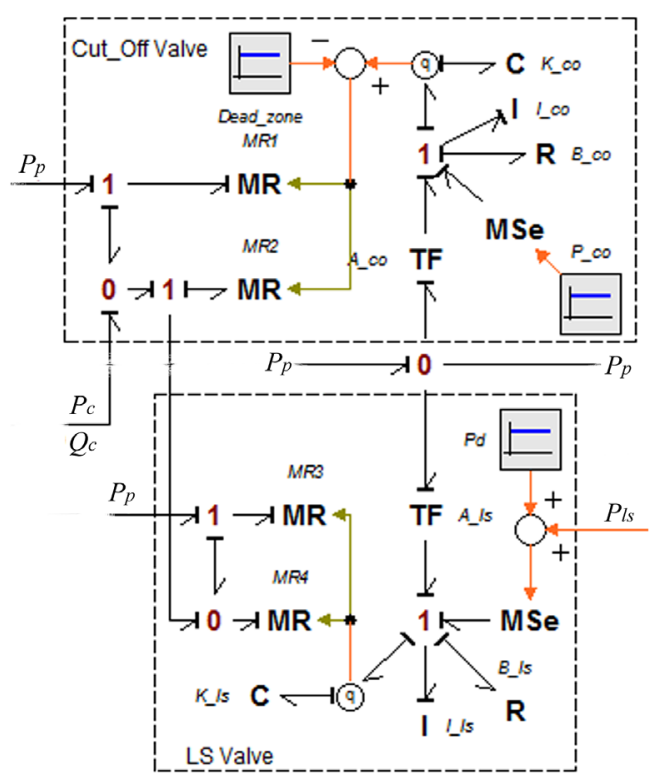

b)

Fig. 4. LS valve and cut-off valve modelling; a) physical structure, b) bond graph model 


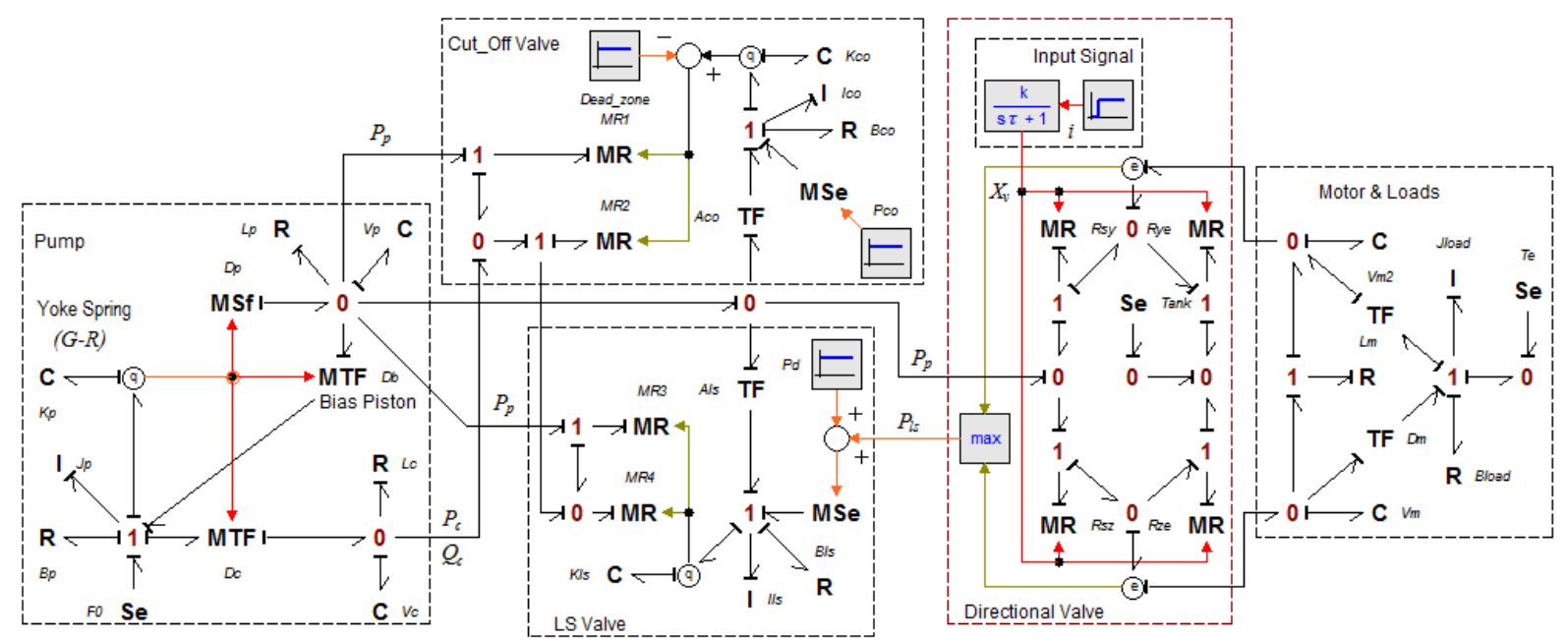

Fig. 5. The full bond graph model of the LS system

\subsection{Simulation and Experimental Validations}

In order to verify the validation of the bond graph model, a series of experiments and computer simulations was performed.
The set-up used in the experiments is shown in Fig. 6. A multifunction data acquisition device, NI USB-6003 (8 AI, 100 kHz; 2 AO, 5 kHz; 1 Counter/ Timer, $5 \mathrm{MHz}$ ) is used for the measurements and the control function with the LabVIEW software on
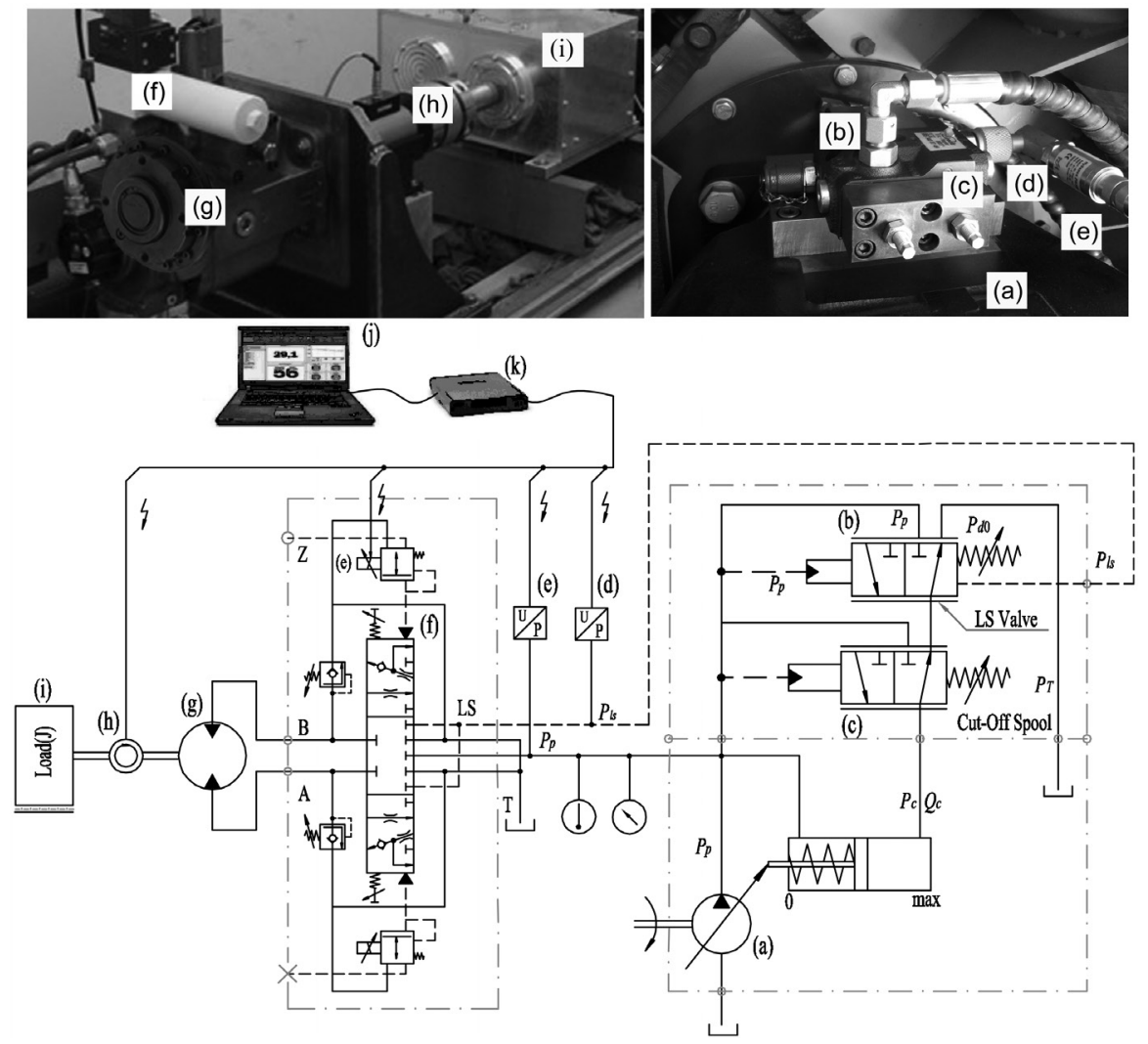

Fig. 6. Experimental set-up: (a) pump, (b) LS valve, (c) cut-off valve, (d) PIs pressure transducer, (e) Pp pressure transducer, (f) directional valve, (g) motor, (h) tachometer, (i) load, (j) computer, (k) DAQ. 
a ThinkPad E445 laptop computer. NI DIAdem is used for the analysis of the measurement results. An LS pump with $20 \mathrm{~cm}^{3} / \mathrm{rev}$ displacement volume is selected, and the speed of the E-motor is $800 \mathrm{rpm}$. A rotating inertial load $\left(J=0.032 \mathrm{~kg} \cdot \mathrm{m}^{2}\right)$ with damping resistance (viscous friction coefficient $B_{\text {load }}=0.03$ $\mathrm{Nm} / \mathrm{rad} / \mathrm{s}$ ) driven by a fixed displacement motor (20 $\mathrm{cm}^{3} / \mathrm{rev}$ ) will be the case to be tested.

As shown in Fig. 6, the pump output pressure and load speed are measured, and one analogue output signal is used for the control of the directional valve. A thermometer is used to measure the fluid temperature, which should be controlled below $60^{\circ} \mathrm{C}$. The measurement results are filtered by an analogue filter with a cut-off frequency of $25 \mathrm{~Hz}$ to remove the high-frequency components.

The main system parameters are listed in Table 1.

Table 1. The main system parameters

\begin{tabular}{lll}
\hline Name & Value & Unit \\
\hline$\beta$ & $6.86 \mathrm{E}+08$ & $\mathrm{~Pa}$ \\
\hline$D_{p}$ & $5.58 \mathrm{E}-03$ & $\left(\mathrm{~m}^{3} / \mathrm{s}\right) / \mathrm{rad}$ \\
\hline$L_{p}$ & $1.58 \mathrm{E}-12$ & $\left(\mathrm{~m}^{3} / \mathrm{s}\right) / \mathrm{Pa}$ \\
\hline$V_{p}$ & $1.70 \mathrm{E}-03$ & $\mathrm{~m}^{3}$ \\
\hline$D_{b}$ & $1.28 \mathrm{E}-05$ & $\mathrm{~m}^{3} / \mathrm{rad}$ \\
\hline$L_{c}$ & $3.51 \mathrm{E}-12$ & $\left(\mathrm{~m}^{3} / \mathrm{s}\right) / \mathrm{Pa}$ \\
\hline$V_{c}$ & $3.03 \mathrm{E}-05$ & $\mathrm{~m}^{3}$ \\
\hline$D_{c}$ & $4.43 \mathrm{E}-05$ & $\mathrm{~m}^{3} / \mathrm{rad}$ \\
\hline$K_{l s}$ & $4.43 \mathrm{E}+04$ & $\mathrm{~N} / \mathrm{m}$ \\
\hline$A_{l s}$ & $3.16 \mathrm{E}-05$ & $\mathrm{~m}^{2}$ \\
\hline$K_{c}$ & $2.48 \mathrm{E}-05$ & $\left(\mathrm{~m}^{3} /(\mathrm{N} 1 / 2 \mathrm{~s})\right.$ \\
\hline$K_{c o}$ & $3.24 \mathrm{E}+03$ & $\mathrm{~N} / \mathrm{m}$ \\
\hline$A_{c o}$ & $5.30 \mathrm{E}-06$ & $\mathrm{~m}^{2}$ \\
\hline$K_{d}$ & $7.45 \mathrm{E}-05$ & $\left(\mathrm{~m}^{3} / \mathrm{s}\right) / \mathrm{m}$ \\
\hline$D_{m}$ & $5.58 \mathrm{E}-03$ & $\left(\mathrm{~m}^{3} / \mathrm{s}\right) / \mathrm{rad}$ \\
\hline$L_{m}$ & $3.88 \mathrm{E}-12$ & $\left(\mathrm{~m}^{3} / \mathrm{s}\right) / \mathrm{Pa}$ \\
\hline$V_{m}$ & $1.83 \mathrm{E}-04$ & $\mathrm{~m}^{3}$ \\
\hline$J$ & 0.032 & $\mathrm{~kg} \cdot \mathrm{m}^{2}$ \\
\hline$T_{e}$ & 0 & $\mathrm{~N} \cdot \mathrm{m}$ \\
\hline$B_{\text {load }}$ & 0.03 & $(\mathrm{Nm} / \mathrm{rad}) / \mathrm{s}$ \\
\hline
\end{tabular}

The initial state of the directional valve opening is fully closed, and the inertial load is standing still. The setting pressure of the cut-off valve is $12 \mathrm{MPa}$; the initial prescribed pressure margin $P_{d 0}$ is $2.8 \mathrm{MPa}$. The simulations are performed on an AMD A10-5750M CPU using the open-source OpenModelica software which supports the bond graph library developed with the Modelica language. The derived mathematical equation is solved by the Backward Differentiation
Formula (BDF) solver, and the tolerance error is 0.0001 .

Two group experiments and simulations are carried out: a) $i=10 \mathrm{~mA}, P_{d}=0.7 \mathrm{MPa}$, b) $i=20 \mathrm{~mA}$, $P_{d}=1.07 \mathrm{MPa}$. The opening step signal of the directional valve is ignited at $1 \mathrm{~s}$.

The dynamic responses of pump delivery pressure $P_{p}$, load pressure $P_{l s}$, load speed and load displacement are selected as the comparison variables of the experiments and simulations.

Fig. 7 shows the comparisons of the selected variables between experimental tests and simulation results with operating conditions $i=10 \mathrm{~mA}$, $P_{d}=0.7 \mathrm{MPa}$. Fig. 8 shows the comparisons with operating conditions $i=20 \mathrm{~mA}, P_{d}=1.07 \mathrm{MPa}$.

As shown in Figs. 7 and 8, both experiments and simulations indicate that, before the input signal is ignited, the LS system is in the idle condition, the pump displacement is de-stroked until the delivery flow is nearly zero, which only complements system leakages and used for heat removal. The pump supplies only a lower pressure which is decided by the prescribed pressure margin $P_{d}$ and the residual pressure of the LS port.

When the input signal is ignited at $1 \mathrm{~s}$, the pump delivery pressure $P_{p}$ increases approximately at a prescribed pressure margin $P_{d}$ greater than the load pressure $P_{l s}$, until rising to $12 \mathrm{MPa}$ then the cut-off valve plays a role. As the load rotates, inspection of the variations of the pump pressure and the load speed shown in Fig. 7, an oscillatory process exists at the beginning, then they become stable after the oscillatory process lasting about 3 seconds, which means that the LS system is stable at the operating conditions $i=10 \mathrm{~mA}, P_{d}=0.7 \mathrm{MPa}$. In Fig. 8 , the oscillatory process will continue, which means that the LS system is in an unstable condition at the operating conditions $i=20 \mathrm{~mA}, P_{d}=1.07 \mathrm{MPa}$. Krus [5] has proven that the instability of the LS system is critically influenced by the mechanical resonance between load dynamic and pump dynamic.

From the comparisons of pump delivery pressure $P_{p}$ and load pressure $P_{l s}$ between experimental tests and simulation results, as shown in Fig. $7 \mathrm{a}$ and $8 \mathrm{a}$, the model, reflects the characteristic of the LS system which supplies "on-demand" flow at a prescribed pressure margin greater than the load pressure of the system. Furthermore, the comparisons of load speed and displacement as shown in Figs. $7 \mathrm{~b}$ and $\mathrm{c}$ and Figs. $8 \mathrm{~b}$ and $\mathrm{c}$, indicate that the full bond graph model is a close representation of the actual LS system at the given operating conditions. 

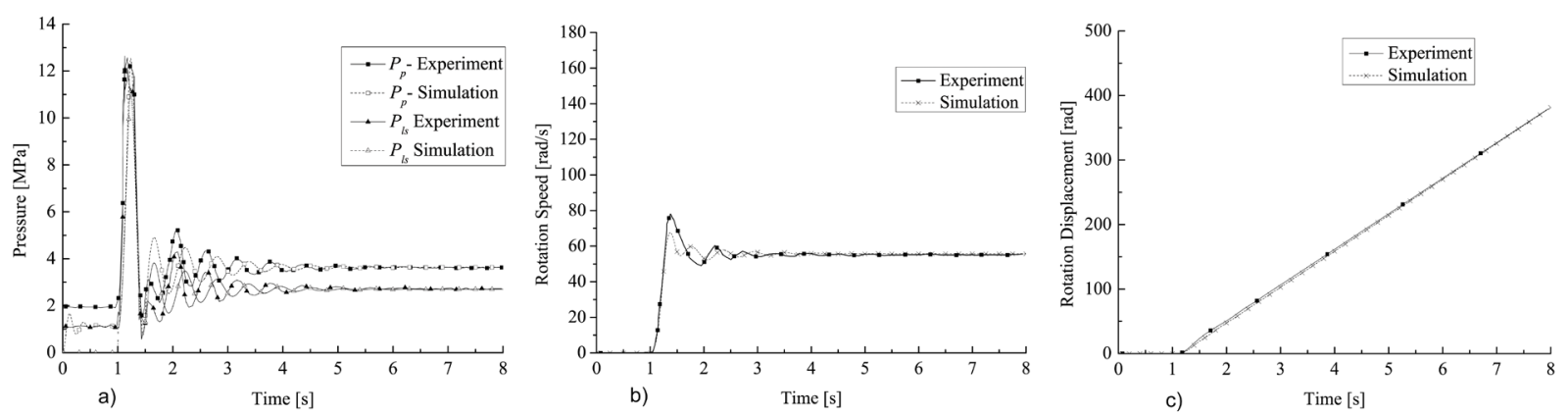

Fig. 7. Comparisons of experimental tests and simulation results ( $\left.i=10 \mathrm{~mA}, P_{d}=0.7 \mathrm{MPa}\right)$; a) pump delivery pressure and load pressure, $b$ ) load speed, c) load displacement
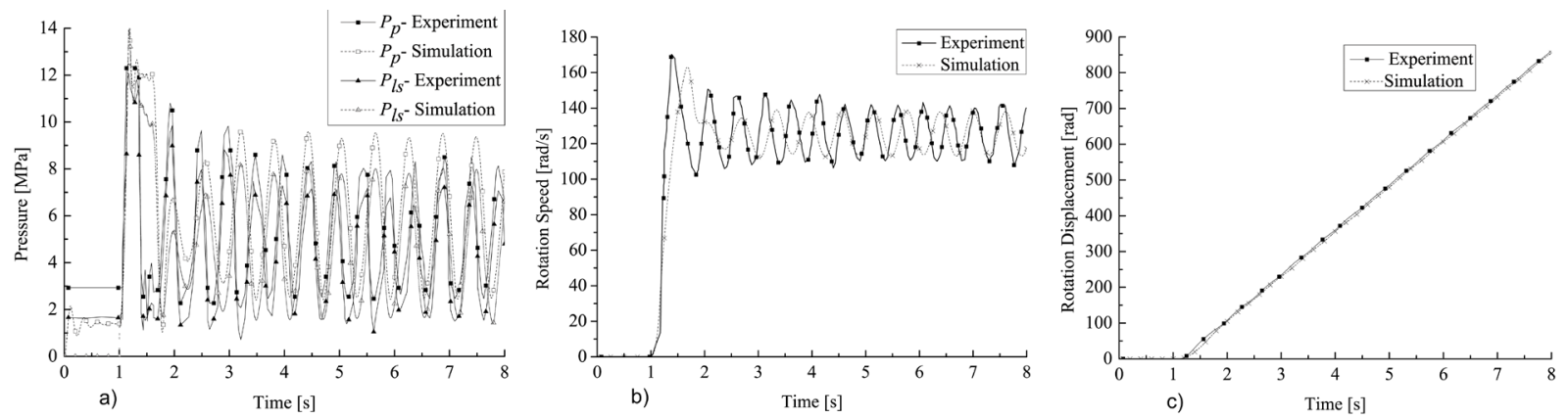

Fig. 8. Comparisons of experimental tests and simulation results ( $i=20 \mathrm{~mA}, P_{d}=1.07 \mathrm{MPa}$ ); a) pump delivery pressure and load pressure, b) load speed, c) load displacement

\section{MODEL REDUCTION BASED ON ACTIVITY INDEX ANALYSIS}

In the previous section, considering the dynamic characteristics of the LS valve and the cut-off valve, an accurate but overly complex bond graph model of the LS system is obtained. In this section, based on activity index analysis, two reduced models with the different threshold are obtained directly from the bond graph level in order to achieve a balance between accuracy and complexity.

\subsection{Theoretical Background}

Rosenberg and Zhou proposed a power-based model reduction algorithm [18] which would lead to instantaneous and time-varying decisions about the importance of elements. Instead of instantaneous power, Louca and Stein [20] proposed the concept of activity, which is defined as the aggregate measure of power flow in or out of an element during the specified time window $T$. The temporal integration of the absolute power value through an element can be expressed as

$$
A_{i}(t)=\int_{t_{0}}^{t_{0}+T}|P(t)| d t=\int_{t_{0}}^{t_{0}+T}|e(t) f(t)| d t,
$$

where is $A_{i}(t)$ the activity of the $i^{\text {th }}$ energy element, $e(t)$, $f(t)$ are generalized effort variable and flow variable in the bond, respectively, and $T$ is the specified time window.

In Eq. (8), the time histories of the effort variables $e(t)$ and flow variables $f(t)$ can be obtained for all elements when simulating the bond graph model.

The activity index of the $i^{\text {th }}$ element in a submodel is the ratio between the activity of the element to be considered and the total activity of the system, and yields as follows:

$$
A I_{i}=\frac{A_{i}}{\sum_{\text {total }} A_{i}} \times 100 .
$$

The core metric for quantifying the relative importance of the elements for a given system based on activity index analysis is that the most important elements are those characterized by the largest magnitudes of the activity index, which have important contributions to the system dynamic. In contrast, the elements with low activity have small contributions. Thus they are unnecessary to the given scenario and can be eliminated from the model to generate a reduced model. 
The procedure of model order reduction algorithm (MORA) based on activity index analysis is shown in Fig. 9.

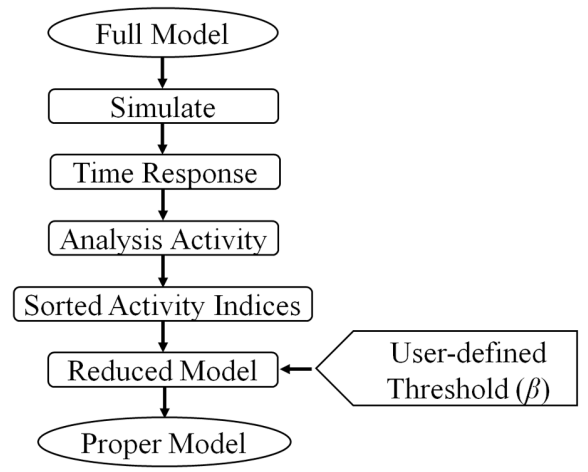

Fig. 9. Procedure of model order reduction algorithm

Firstly, the model is simulated, and the time response of the variables in Eq. (8) is available for the activity computation. Then, the activity index for each energy storage and dissipation element is calculated by Eq. (9). Finally, the activity indices are sorted in descending order, the result of the sorting process is the ranking of the element importance. Based on a user-defined threshold $\beta$ for a specific scenario, the surplus elements with low activity index can be identified and eliminated directly from the bond graph model while retaining sufficiently accurate predictions.

The activity index analysis is an energy-based model reduction method; it is especially suitable for the bond graph models due to the common energybased nature. Moreover, by analysing the activity index, the model can be simplified directly at the bond graph level.

\subsection{Reduced Model}

A full bond graph model of the LS system has been constructed, as displayed in Fig. 5, the model has 26 energy storage and dissipation elements, and it is an eleventh-order dynamic system.

The model simulations are carried out with the operating conditions $P_{d}=1.07 \mathrm{MPa}$ and two different input signals, $i=20 \mathrm{~mA}$ and $i=5 \mathrm{~mA}$, the time window $T$ is from $0 \mathrm{~s}$ to $8 \mathrm{~s}$, and the user-defined threshold is $\beta=99.5$. The differential equation is solved by the $\mathrm{BDF}$ solver, and the tolerance error is 0.0001 .

The activity index of all the energy storage and dissipation elements are calculated, and the activity indices are sorted according to $i=20 \mathrm{~mA}$ groups in descending order. The result is listed in Table 2 .
By setting the threshold $\beta=99.5$, the elements with low activity index, which are less important and can be eliminated from the bond graph model with few effects on the system dynamics, are identified and enclosed in a grey, as shown in Table 2.

Table 2. Sorting of the activity index

\begin{tabular}{|c|c|c|c|c|}
\hline \multirow{2}{*}{$\begin{array}{l}\text { Energy } \\
\text { Element }\end{array}$} & \multicolumn{2}{|c|}{$i=20 \mathrm{~mA}$} & \multicolumn{2}{|c|}{$i=5 \mathrm{~mA}$} \\
\hline & Activity & $\mathrm{Al}$ & Activity & $\mathrm{Al}$ \\
\hline$L_{p}$ & 5247.33 & 29.43 & 1825.6 & 50.67 \\
\hline$B_{\text {load }}$ & 3245.57 & 18.20 & 203.46 & 5.65 \\
\hline$V_{p}$ & 2564.83 & 14.38 & 429.52 & 11.92 \\
\hline$J_{\text {load }}$ & 2420.61 & 13.58 & 62.74 & 1.74 \\
\hline$R_{y e}$ & 1591.00 & 8.92 & 394.11 & 10.94 \\
\hline$R_{S z}$ & 1578.84 & 8.85 & 390.47 & 10.84 \\
\hline$M R_{3}$ & 426.47 & 2.39 & 141.37 & 3.92 \\
\hline$L_{m}$ & 230.18 & 1.29 & 18.29 & 0.51 \\
\hline$L_{c}$ & 164.04 & 0.92 & 80.17 & 2.23 \\
\hline$V_{m 1}$ & 117.59 & 0.66 & 14.85 & 0.41 \\
\hline$M R_{1}$ & 79.35 & 0.45 & 0.00 & 0.00 \\
\hline$M R_{2}$ & 74.59 & 0.42 & 18.78 & 0.52 \\
\hline$V_{c}$ & 30.94 & 0.17 & 1.24 & 0.03 \\
\hline$M R_{4}$ & 25.910 & 0.145 & 5.686 & 0.158 \\
\hline$K_{c o}$ & 15.350 & 0.086 & 11.493 & 0.319 \\
\hline$J_{p}$ & 6.804 & 0.038 & 0.216 & 0.006 \\
\hline$V_{m 2}$ & 6.301 & 0.035 & 1.553 & 0.043 \\
\hline$I_{c o}$ & 2.695 & 0.015 & 2.694 & 0.075 \\
\hline$K_{l s}$ & 0.860 & 0.005 & 0.258 & 0.007 \\
\hline$K_{p}$ & 0.512 & 0.003 & 0.059 & 0.002 \\
\hline$B_{c o}$ & 0.368 & 0.002 & 0.365 & 0.010 \\
\hline$I_{l s}$ & 0.113 & 0.001 & 0.042 & 0.001 \\
\hline$B_{p}$ & 0.106 & 0.001 & 0.015 & 0.000 \\
\hline$B_{l s}$ & 0.005 & 0.000 & 0.002 & 0.000 \\
\hline$R_{z e}$ & 0.000 & 0.000 & 0.000 & 0.000 \\
\hline$R_{s v}$ & 0.000 & 0.000 & 0.000 & 0.000 \\
\hline
\end{tabular}

The components in gray include the inertia, resistance, and capacitance effects of the LS valve, the cut-off valve and the motion dynamic of the swash-plate, respectively, which have smaller contributions to the system dynamics. It proves the rationality representing the LS pump dynamics with a fourth-order differential equation and neglecting the dynamics of the LS valve and the cut-off valve in the literature. Furthermore, the leakage of the pump and motor play important roles in the system dynamics due to its correlation to the damping coefficient.

When determining the eliminated elements among the enclosed elements, $M R_{4}, R_{z e}$ and $R_{s y}$ are retained in order to keep the bond graph structure in accordance with the topologic structure of the real LS 


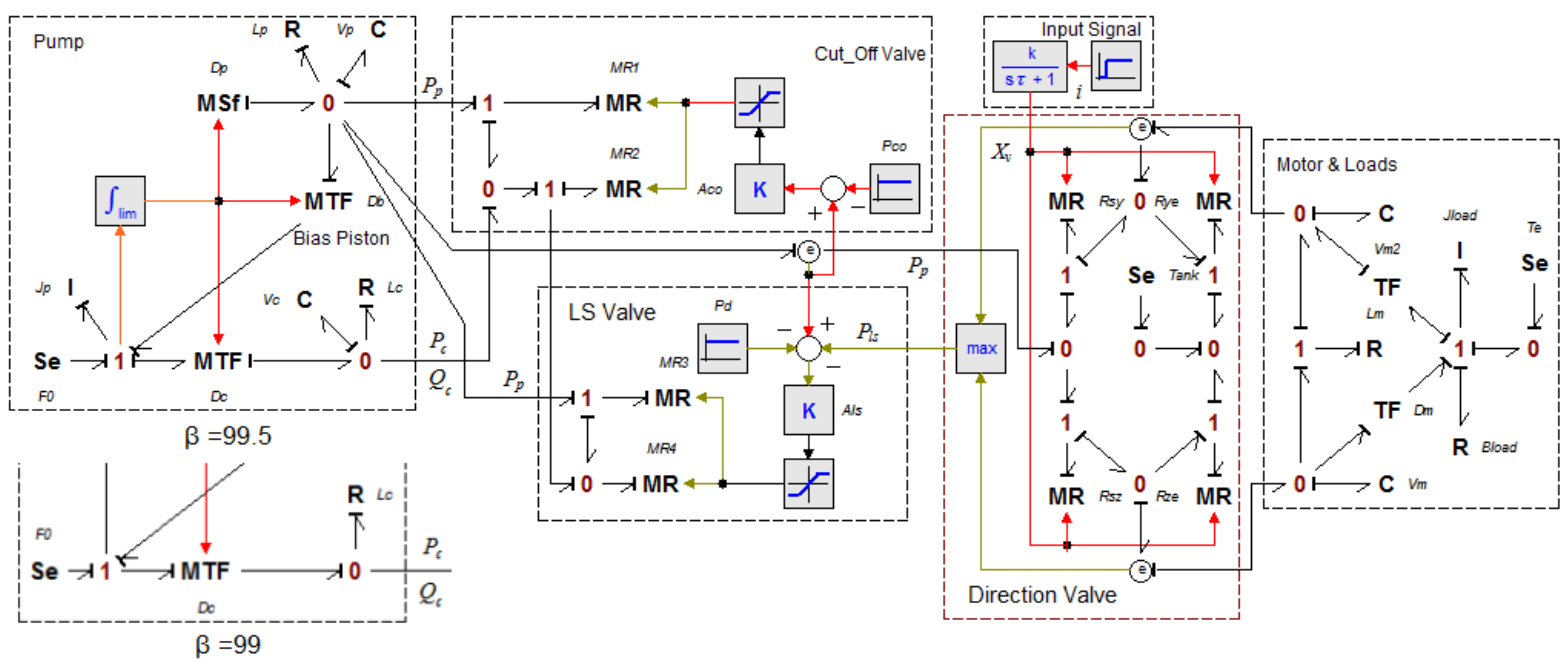

Fig. 10. The reduced $L S$ system with threshold $\beta=99.5$ and $\beta=99$

system. $J_{p}$ is also retained in order to eliminate the derivative causality assigned to the $V_{c}$ capacitance element which implies the difficulty when solving the differential algebraic equations (DAEs). The elements can be eliminated directly at the bond graph level. Thus, the reduced model with threshold $\beta=99.5$ can be obtained, as shown in Fig. 10.

Now turning to the low activity indices of $i=5$ $\mathrm{mA}$, as shown in Table 2 , there are intersections of $\left\{B_{p}\right.$ $\left.B_{l s} I_{l s} B_{c o} K_{l s} I_{c o} K_{c o}\right\}$ to be eliminated among the three different operating conditions. This indicates that the reduced bond graph model with threshold $\beta=99.5$ is suitable for the input signal range of $i=5 \mathrm{~mA}$ to 20 $\mathrm{mA}$.

Also by setting the threshold $\beta=99$, the elements $M R_{1}, M R_{2}$ and $V_{c}$ can be eliminated. For the same reason preserving the bond graph structure, $M R_{1}$, $M R_{2}$ are retained, and $V_{c}$ is eliminated, because there is no derivative causality assigned to $V_{c}$, so $J_{p}$ can be eliminated. Thus the intersections of $\left\{B_{p} J_{p} V_{c} B_{l s} I_{l s} K_{l s}\right.$ $\left.B_{c o} I_{c o} K_{c o}\right\}$ to be eliminated among the three different operating conditions are listed, and the reduced model with threshold $\beta=99$ is available. Compared to the reduced model with threshold $\beta=99.5$, the model is almost the same; in addition, $J_{p}$ and $V_{c}$ are eliminated, as shown in the left corner of Fig. 10.

\subsection{Accuracy Assessment and Discussions}

To assess the accuracy quantitatively, the average relative error $\varepsilon$ is calculated using Eq. (10):

$$
\varepsilon=\frac{\int_{t_{0}}^{t_{0}+T}\left|\omega(t)-\omega_{r}(t)\right| d t}{\int_{t_{0}}^{t_{0}+T}|\omega(t)| d t} \times 100
$$

where $\omega(t)$ is the response of evaluated variable in the full model, and $\omega_{r}(t)$ is the response of evaluated variable in the reduced model.

In order to assess the accuracy of the reduced bond graph model of the LS system, the important state variables that are easy to measure are selected as the evaluation metrics. In the paper, the dynamic responses of pump delivery pressure $P_{p}$, load pressure $P_{l s}$, load speed and load displacement are selected as the comparison variables of the full model and the reduced model with threshold $\beta=99.5$ and $\beta=99$, respectively.

Using Eq. (10), the average relative error of pump delivery pressure $P_{p}$, load pressure $P_{l s}$, load speed and load displacement respectively is $1.27 \%$, $1.25 \%, 0.3 \%$, and $0.13 \%$, when setting threshold $\beta=99.5$. While the average relative error respectively is $27.3 \%, 25.17 \%, 5.34 \%$, and $0.49 \%$, when setting threshold $\beta=99$. The results are shown in Table 3 .

In order to visualize the comparisons, the dynamic responses and relative errors of the delivery pressure $P_{p}$, load pressure $P_{l s}$, load speed and load displacement of the full model and the reduced model with threshold $\beta=99.5$ and $\beta=99$ are compared, the results are shown in Fig. 11. 

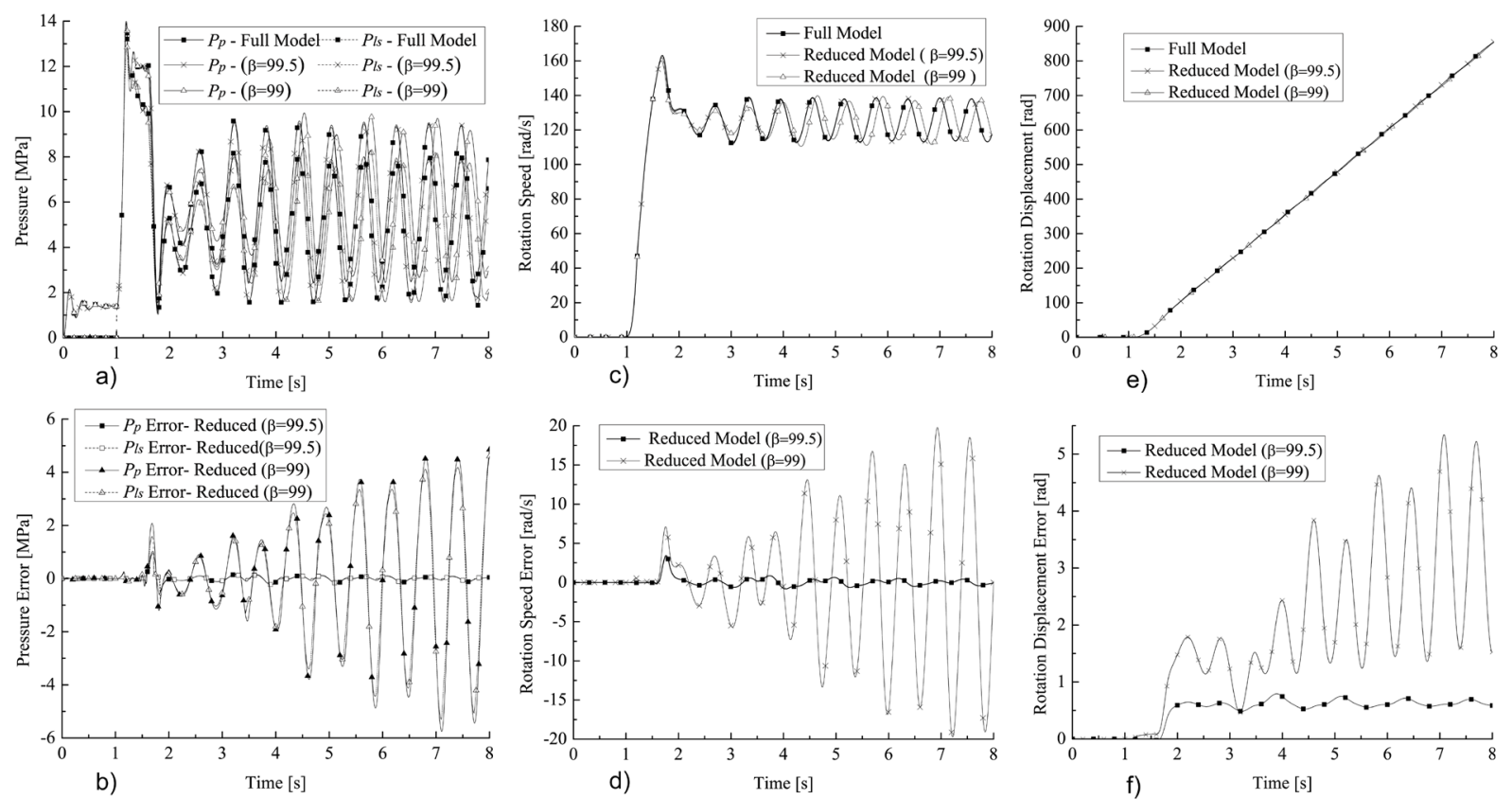

Fig. 11. Comparisons of full model and reduced models; a) pump delivery pressure and load pressure, b) pressure errors, c) load speed response, d) speed errors, e) load-displacement response, f) displacement errors

Table 3. Accuracy level of reduced model [\%]

\begin{tabular}{lccccc}
\hline$\beta$ & \multirow{2}{*}{ Eliminated Element } & $P_{p}$ & $P_{l s}$ & Speed & Disp. \\
& & Error & Error & Error & Error \\
\hline 99.5 & $B_{l s} B_{p} I_{l s} B_{c o} K_{l s} I_{c o} K_{c o}$ & 1.27 & 1.25 & 0.30 & 0.13 \\
\hline 99 & $B_{l s} B_{p} I_{l s} B_{c o} K_{l s} I_{c o} J_{p} K_{c o} V_{c}$ & 27.26 & 25.17 & 5.34 & 0.49 \\
\hline
\end{tabular}

As shown in Table 2, by analysing the activity index, it can be found out that the leakage of the pump and the viscous friction coefficients of the load are the most important parameters to the system dynamics.

Table 4 shows the system order and the computational load in seconds of the full model, and the reduced models with threshold $\beta=99.5$ and $\beta=99$, respectively.

Table 4. Accuracy level of reduced model

\begin{tabular}{lcc}
\hline Model & System order & Computation time [s] \\
\hline Full & 11 & 3.225 \\
\hline$\beta=99.5$ & 7 & 2.830 \\
\hline$\beta=99$ & 4 & 2.667 \\
\hline
\end{tabular}

\section{CONCLUSIONS}

Based on the bond graph methodology, an accurate but overly complex eleventh-order bond graph model of the LS system model is constructed, which takes into account the dynamic characteristics of the LS valve and the cut-off valve. A series of experiments are performed to verify the validation of the model, the results of which indicate that the full bond graph model is a close representation of the actual LS system at given operating conditions. Furthermore, compared to the simplified abstract mathematical models, the bond graph model preserves the computational structure and the topological structure of the LS system, and it is convenient to modify the model directly at the graphical representation level.

To simplify the full model of the LS system to achieve a balance between accuracy and complexity, the model reduction technology based on the activity index analysis is used, and two reduced models with threshold $\beta=99.5$ and $\beta=99$ are obtained. The relative error of pump delivery pressure, load pressure, load speed and load displacement is $1.27 \%, 1.25 \%$, $0.3 \%$, and $0.13 \%$, respectively, when setting the threshold $\beta=99.5$. While the relative error is $27.3 \%$, $25.17 \%, 5.34 \%$, and $0.49 \%$, respectively, when setting threshold $\beta=99$. Meanwhile, the order of the full system is decreased from eleventh-order to seventh-order and fourth-order respectively, and the computation time is reduced from $3.714 \mathrm{~s}$ to $3.236 \mathrm{~s}$ and $2.667 \mathrm{~s}$, respectively. Results indicate that the reduced model with threshold $\beta=99.5$ have almost identical predictions for system dynamics. 
Furthermore, by quantifying the relative importance of the elements of the LS system based on activity index analysis, it proves the rationality representing the LS pump dynamics with a fourthorder differential equation, and neglecting the dynamics of the LS valve and the cut-off valve in the literature, also the leakage of the pump plays an important role in the system dynamics due to its correlation to the damping coefficient of the LS system.

\section{ACKNOWLEDGEMENTS}

The authors gratefully acknowledge the support by the National Natural Science Foundation of China through the grant number 51275068 .

\section{NOMENCLATURES}

$B$ equivalent bulk modulus, $[\mathrm{Pa}]$

$D_{p}$ equivalent bulk modulus of pump, $\left[\left(\mathrm{m}^{3} / \mathrm{s}\right) / \mathrm{rad}\right]$

$L_{p} \quad$ leakage coefficient of the pump, $\left[\left(\mathrm{m}^{3} / \mathrm{s}\right) / \mathrm{Pa}\right]$

$V_{p}$ volume in pump chamber and the line, $\left[\mathrm{m}^{3}\right]$

$D_{b} \quad$ volumetric displacement of bias piston, $\left[\mathrm{m}^{3} / \mathrm{rad}\right]$

Lc leakage coefficient of control piston, $\left[\left(\mathrm{m}^{3} / \mathrm{s}\right) / \mathrm{Pa}\right]$

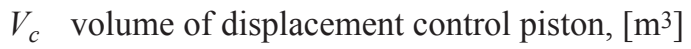

$D_{c}$ volumetric displacement of control cylinder, $\left[\mathrm{m}^{3} / \mathrm{rad}\right]$

$G$ coefficient of displacement control spring, $[\mathrm{Nm} / \mathrm{rad}]$

$\mathrm{R}$ inertia force of piston and attachments, [Nm/rad]

$K_{l s}$ coefficient of the LS spring, [N/m]

$A_{l s}$ area of the LS valve piston [m²]

$K_{c}$ flow coefficient of LS \& cut-off valve, $\left[\mathrm{m}^{3} /\left(\mathrm{N}^{1 / 2} \mathrm{~s}\right)\right]$

$K_{c o}$ coefficient of cut-off valve spring, [N/m]

$A_{c o}$ spool area of cut-off valve, $\left[\mathrm{m}^{2}\right]$

$K$ gain of spool displacement [m/A]

$\tau$ time constant of the reduce valve, [s]

$K_{d}$ flow coefficient of direction valve, $\left[\left(\mathrm{m}^{3} / \mathrm{s}\right) / \mathrm{m}\right]$

$D_{m}$ volumetric displacement of motor, $\left[\left(\mathrm{m}^{3} / \mathrm{s}\right) / \mathrm{rad}\right]$

$L_{m}$ leakage coefficient of motor, $\left[\left(\mathrm{m}^{3} / \mathrm{s}\right) / \mathrm{Pa}\right]$

$V_{m}$ volume in motor chamber and the line, $\left[\mathrm{m}^{3}\right]$

$J$ moment inertia of motor and loads, $\left[\mathrm{kg} \cdot \mathrm{m}^{2}\right]$

$T_{e}$ external torque, $[\mathrm{N} \cdot \mathrm{m}]$

$B_{\text {load }}$ viscous friction coefficient of the load, $[(\mathrm{Nm} / \mathrm{rad}) / \mathrm{s}]$

\section{REFERENCES}

[1] Xu, B., Cheng M., Yang H.Y. (2012). A new electrohydraulic load sensing control system for hydraulic excavators. Proceedings of the 8th International Fluid Power Conference vol. 1, p. 553565.

[2] Carpena, A.G., Hijes, F.C.G.D.E., García, M.A., Moreno, F.M.M. (2015). Checking load sensing valves at vehicle inspections stations. Mechanics Industry, vol. 16, no. 3, p. D0I:10.1051/ meca/2015006.

[3] Backe, W., Zahe, B. (1993). Electrohydraulic loadsensing. Research Projects in Hydraulics. Fluid Power, p. 1-27.

[4] Kim, S.D., Cho, H.S. (1991). A suboptimal controller-design method for the energy efficiency of a load-sensing hydraulic servo system. Journal of Dynamic Systems, Measurement, and Control, vol. 113, no. 3, p. 487-493, Dol:10.1115/1.2896436.

[5] Krus, P. (1988). On Load Sensing Fluid Power Systems. Ph.D thesis, no. 198. Linkoping University, Linkoping.

[6] Jayaraman, G.P., Lunzman, S.V. (2011). Modeling and analysis of an electronic load sensing pump. IEEE International Conference on Control Applications, Dol:10.1109/ CCA.2011.6044497.

[7] Axin, M., Eriksson, B., Krus, P. (2014). Flow versus pressure control of pumps in mobile hydraulic systems. Proceedings of the Institution of Mechanical Engineers, Part I: Journal of Systems and Control Engineering, vol. 228, no. 4, p. 245-56, DOl:10.1177/0959651813512820.

[8] Xu, B., Cheng, M., Yang, H.Y. (2015). A hybrid displacement/ pressure control scheme for an electrohydraulic flow matching system. IEEE/ASME Transactions on Mechatronics, vol. 20, no. 6, p. 2771-2782, DOI:10.1109/TMECH.2015.2411315.

[9] Koivumaki, J., Mattila, J. (2014). Stable and high performance energy-efficient motion control of electric load sensing controlled hydraulic manipulators. Proceedings of the Asme/ Bath Symposium on Fluid Power and Motion Control, Sarasota.

[10] Wagner, Z.D, Fales, R. (2014). Modeling and stability of a hydraulic load-sensing pump with investigation of a hard nonlinearity in the pump displacement control system. Proceedings of the Asme/Bath Symposium on Fluid Power and Motion Control, Bath, DOl:10.1115/FPMC2014-7802.

[11] Wu, D. (2003). Modeling and Experimental Evaluation of a Load-Sensing and Pressure Compensated Hydraulic System, Ph.D. thesis, University of Saskatchewan, Saskatchewan.

[12] Sakurai, Y. (2000). Calculation of dynamic overall efficiency of a load sensing hydraulic system by bondgraphs. $26^{\text {th }}$ Annual Confjerence of the IEEE Industrial Electronics Society, vol. 3, p. 1568-1573, DOI:10.1109/iecon.2000.972508.

[13] Sakurai, Y., Nakada, T., Tanaka, K. (2002). Design method of an intelligent oil-hydraulic system (load sensing oilhydraulic system). Proceedings of the IEEE International Symposium on Intelligent Control, p. 626-630, D0l:10.1109/ ISIC.2002.1157835.

[14] Karnopp, D.C., Margolis, D.L., Rosenberg, R.C. (2012). System Dynamics: Modeling, Simulation, and Control of Mechatronic Systems, 5th rd., John Wiley \& Sons, New York, DOl:10.1002/9781118152812.

[15] Borutzky, W. (2010). Bond Graph Methodology - Development and Analysis of Multidisciplinary Dynamic System Models. Springer, London.

[16] Tian, S.J, Hu, Q.Y, Zhang, H. (2012). Dynamic Simulation of Hydraulic Systems, $2^{\text {nd }}$ ed. Dalian University of Technology Press, Dalian. (in Chinese) 
[17] Ersal, T., Fathy, H.K., Rideout, D.G., Louca, L.S., Stein, J.L. (2008). A review of proper modeling techniques. Journal of Dynamic Systems, Measurement, and Control, vol. 130, no. 6, DOI:10.1115/1.2977484.

[18] Rosenberg, R.C., Zhou, T. (1988). Power-Based Simplification of Dynamic System Models. Proceedings of Advances in Design Automation, Kissimmee, vol. 14, p. 487-492.

[19] Louca, L.S., Stein, J.L., Hulbert, G.M. (2010). Energy-Based Model Reduction Methodology for Automated Modeling. Journal of Dynamic Systems Measurement and Control, vol.132, no. 6, Dol:10.1115/1.4002473.
[20] Louca, L.S., Stein, J.L. (1998). Physical interpretation of reduced bond graphs. Proceedings of $2^{\text {nd }}$ IMACS International Multiconference: Computational Engineering in Systems and Applications, Nabeul-Hammamet.

[21] Ivantysyn, J., Ivantysynova, M. (2001). Hydrostatic Pumps and Motors: Principles, Design, Performance, Modeling, Analysis, Control and Testing. India Akademic Books International, New Delhi.

[22] Manring, N.D. (2013). Fluid Power Pumps and Motors: Analysis, Design and Control, McGraw-Hill, New York. 